\title{
Five-year survival of 3-unit fiber-reinforced composite fixed partial dentures in the posterior area
}

\author{
Céleste C.M. van Heumen ${ }^{a, *}$, Johanna Tanner ${ }^{b}$, Jan W.V. van Dijken ${ }^{c}$, Ronald Pikaar ${ }^{d}$, \\ Lippo V.J. Lassila ${ }^{a}$, Nico H.J. Creugers ${ }^{b}$, Pekka K. Vallittu ${ }^{e}$, Cees M. Kreulen ${ }^{b}$ \\ a Department of Prosthodontics and Biomaterials Science, Institute of Dentistry, University of Turku, Finland \\ ${ }^{\mathrm{b}}$ Department of Oral Function and Prosthetic Dentistry, College of Dental Science, University Medical Centre Nijmegen, The Netherlands \\ c Department of Odontology, Dental School Umeå, Umeå University, Sweden \\ d Centre of Special Dental Care, Rijnstate Medical Centre, Arnhem, The Netherlands \\ e Department of Prosthetic Dentistry and Biomaterials Science, Institute of Dentistry, University of Turku, Finland
}

\section{A R T I C L E I N F O}

Article history:

Received 19 October 2009

Received in revised form

29 January 2010

Accepted 25 May 2010

\section{Keywords:}

Dental restorations

Fiber-reinforced composites

Fixed partial denture

Resin bonded bridge

\begin{abstract}
A B S T R A C T
Objectives. The purpose of this clinical study was to evaluate the long-term outcome of threeunit posterior fixed partial dentures (FPDs) made of fiber-reinforced resin composite (FRC), and to identify design factors influencing the survival rate.

Methods. 77 patients (52 females, 25 males) received 96 indirectly made FRC FPDs, using preimpregnated unidirectional glass-fibers, requiring manual wetting, as framework material. FPDs were surface $(n=31)$ inlay $(n=45)$ or hybrid $(n=20)$ retained and mainly located in the upper jaw. Hybrid FPDs consisted of a wing retainer at canine and an inlay retainer at distal abutment tooth. Surface FPDs consisted of uplay and wing combinations. Follow-up period was at minimum 4.5 years, with checkups at every 1-2 years. The study was carried out by six operators in three centers in the Netherlands, Finland and Sweden. Survival rates, including reparable defects of FPDs, and success rates were determined.

Results. Kaplan-Meier survival rate at 5 years was $71.2 \%$ (SE $4.8 \%$ ) for success and $77.5 \%$ (SE 4.4\%) for survival. Differences were not significantly different. Main failure modes were delamination and fracture of the FPD. Only FPDs with surface retainers showed debonding. Conclusions. A success rate of $71 \%$ and a survival rate of $78 \%$ after 5 years was found. Survival rates of inlay, hybrid and surface retained FPDs did not significantly differ.
\end{abstract}

c 2010 Academy of Dental Materials. Published by Elsevier Ltd. All rights reserved.

\section{Introduction}

The fixed partial denture (FPD) is a treatment modality offering tooth tissue conservation together with lower treatment costs. In a recent meta-analysis, the resin bonded FPD fabricated with a metal framework showed an estimated survival rate of $87.7 \%$ after 5 years [1]. Complications like debonding of the framework from the luting cement were frequent and have been related to the unsatisfactory surface treatment of the metal alloy, due to difference in thermal expansion with regard to resin composite luting cements and the rigidity of the metal framework [2]. Moreover, esthetic considerations may be a drawback. It is expected that fiber-reinforced composite

DOI of original article:10.1016/j.dental.2009.01.103.

* Corresponding author at: Department of Oral Function and Prosthetic Dentistry, College of Dental Science, University Medical Centre Nijmegen, PO Box 9101, 6500 HB Nijmegen, The Netherlands. Tel.: +31 24 3614004; fax: +31 243541971.

E-mail address: c.vanheumen@dent.umcn.nl (C.C.M. van Heumen).

0109-5641/\$ - see front matter @ 2010 Academy of Dental Materials. Published by Elsevier Ltd. All rights reserved.

doi:10.1016/j.dental.2010.05.010 
(FRC) FPDs may provide an improved adhesive performance, because the material of the construction is similar to the luting material and FRC constructions are less rigid.

FRCs have recently been developed for dental applications and various types of fibers and fiber-products have been tested as reinforcing materials. Glass fibers are most often used because of their ability to withstand tensile stress and to prevent crack propagation in resin composite materials, and their esthetic character $[3,4]$. Substantial improvements in flexural strength, fracture toughness and elastic modulus have been achieved in dental resin composites reinforced with fibers [5]. The development of fiber products available for dental use has led from plain fibers to pre-impregnated fibers and finally fully resin impregnated fibers.

The retainer designs of an FRC prosthesis can be either fullcoverage or partial coverage types, depending on the condition and amount of remaining sound tissue of the abutment teeth. The freedom in design of the FPD allows a tooth-conserving preparation when the abutment teeth are unrestored or have modest restorations. Fibers in the bridge construction run from the retainer at one end to the other, are preferably located in the tension side of the bridge and are completely covered by resin composite material. In addition, an FRC FPD can be fabricated either directly in the mouth or indirectly by a dental technician.

Two systematic reviews of all commercially available FRC products without discrimination between type of retainers or fabrication technique have been published [6,7]. In both studies a limited number of published clinical studies was found, all of relatively limited duration, and few of the reported commercial products demonstrated robust clinical documentation to support their use. Problems specifically associated with a commonly used system include fractures of the veneering composite [8-10], but also wear [8] and discoloration [10] have been observed. Consequently, there is a need for data on other systems, preferably based on trials of longer duration.

In a recent study we reported 5-year follow-up data of three-unit anterior FRC FPDs, made of manually resin impregnated glass-FRC, which were placed in three academic centers in Finland, the Netherlands and Sweden [11]. The purpose of the present study was to evaluate the long-term clinical outcome of three-unit FRC FPDs, but now applied in the posterior area. The FRC material was identical and all FPDs were indirectly made. Minimum service time was 4.5 years and design factors influencing survival were identified. Studies on metal resin-bonded FPDs showed lower survival rates in the posterior than in the anterior region, thus we expect that the survival rate of FRC FPDs shows the same difference.

\section{Materials and methods}

Between April 1998 and September 2002, 77 patients (52 females, 25 males) of the departments of Oral Function and the Centre of Special Dental Care of the Radboud University of Nijmegen (the Netherlands), the Institute of Dentistry University of Turku (Finland) and the Dental School Umeå (Sweden) were treated with 96 three-unit posterior indirect FRC FPDs. Approval of the University Medical Ethical Committee was obtained (the joint commission on the ethics of the Turku Uni-
Table 1 - Distribution of posterior FRC FPDs $(n=96)$.

\begin{tabular}{lll} 
Variable & & $n$ \\
\hline Jaw & Maxilla & 47 \\
Gender of the patiënt & Male & 46 \\
& Female & 33 \\
Pontic type & Premolar & 60 \\
& Molar & 70 \\
Operator & 2 & 23 \\
& 3 & 21 \\
& 4 & 29 \\
& 5 & 14 \\
Academic center & 6 & 10 \\
& Nijmegen & 19 \\
Material & Turku & 50 \\
& Umeå & 29 \\
Luting cement & Artglass & 14 \\
& Sinfony & 50 \\
& Panavia & 43 \\
& Twinlook & 29 \\
& Compolute & 21 \\
& & 43 \\
\hline
\end{tabular}

versity and the Turku University Central Hospital, Resolution No. 264). Informed consent was given for each patient. The patients' ages ranged from 12.4 to 77.5 years, with a mean age of 38.6 years. All FPDs replaced one missing tooth, which could be the first and second premolar or the first molar, and two adjacent abutment teeth were used for retention. No cantilever bridges were involved. Sixty-two patients received one FPD, thirteen patients received two FPDs and two patients received four FPDs. Among these, it concerned an FPD that was made after the first failed in four cases and these FPDs were included as new cases. The characteristics of the dentitions of subjects and FPDs made are presented in Table 1. Patients were free of extensive periodontal disease and most of them had complete dental arches (except the missing tooth). X-rays to exclude periapical disease and loss of periodontal support of the abutment teeth were available.

We aimed for conservation of tooth tissue and the FPD designs used depended on the level of restoration of the individual abutment teeth. The retainer designs of the two abutment teeth made can be divided into three categories: (1) uplay and wing combinations (surface retained), (2) both inlay retainers (inlay retained), and (3) wing retainer at palatal side of canine, inlay at distal abutment tooth (hybrid FPD) (Fig. 1). Wing retainers (or so-called Maryland design surface retainers) were always provided with occlusal support. This could be designed as a minimal inlay-box preparation or, if there was any interocclusal space, without removal of tooth material. Uplay retainers were designed as an occlusal 'wing'. In 12 cases the inlay retainer was provided with an additional wing at the buccal or lingual surface. The numbers of different FPD designs are described in Table 2. Types of FPD designs were not evenly distributed between the three centers, with most of the surface retained FPDs made in Nijmegen, whereas the material of Turku and Umeå was predominantly of the inlay-type. 
(A)

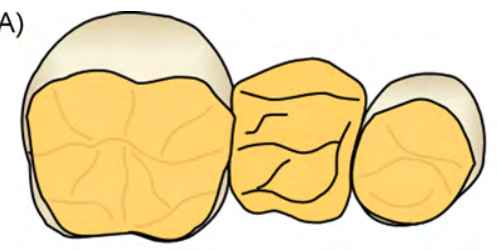

(C)

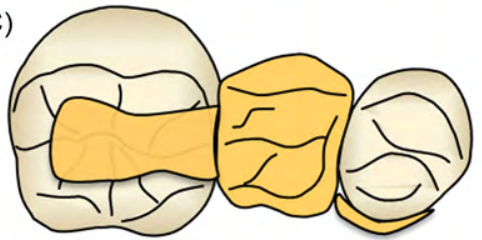

(B)

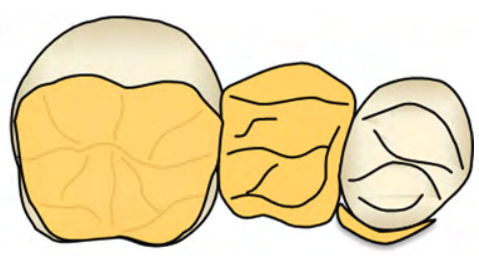

(D)

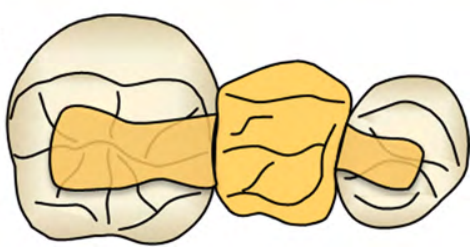

Fig. 1 - Type of FPD: (A) surface retained FPD with uplay retainers at both abutment teeth, (B) surface retained FPD with uplay retainer at distal abutment tooth and wing retainer at mesial abutment tooth, (C) hybrid retained FPD with inlay retainer at distal abutment tooth and wing retainer at mesial abutment tooth, and (D) inlay retained FPD with inlay retainers at both abutment teeth.

Table 2 - Retainer characteristics of surface, inlay and hybrid retained FPDs.

\begin{tabular}{|c|c|c|c|c|c|c|c|}
\hline Type of FPD & Mesial retainer & Distal retainer & $n$ & \multicolumn{2}{|c|}{ Pontic type } & \multicolumn{2}{|c|}{ Jaw } \\
\hline Surface retained & Uplay & Uplay & 15 & Premolar 14 & Molar 1 & Mandibula 11 & Maxilla 4 \\
\hline Surface retained & Wing & Uplay & 16 & Premolar 16 & Molar 0 & Mandibula 10 & Maxilla 6 \\
\hline Hybrid retained & Wing & Inlay & 20 & Premolar 20 & Molar 0 & Mandibula 6 & Maxilla 14 \\
\hline Inlay retained & Inlay & Inlay & 45 & Premolar 25 & Molar 20 & Mandibula 18 & Maxilla 27 \\
\hline
\end{tabular}

\subsection{Restorations}

Treatment was performed by six experienced dentists, with adequate skills in adhesive techniques, according to a clinical protocol. Clinical procedures were performed during two treatment sessions: (1) tooth preparation, impressions and provisional restorations; and (2) try-in, placement of the FPD, and finishing. Tooth preparation involved removal of existing restorations and creating cavities with slight divergence of cavity walls and rounded angles. Inlay-cavities required adequate volume and support for the FRC substructure, at minimum $2 \mathrm{~mm} \times 2 \mathrm{~mm} \times 2 \mathrm{~mm}$ in size. Surface retainers with a minimal thickness of $0.4 \mathrm{~mm}$ at the canines were provided with palatal slots and distal grooves depending on the preference of the operator. Uplay retainers were made without tooth preparation in case occlusal space was available.

Impressions were made with a polyvinyl siloxane material. If present, cavities were protected with a provisional filling material for the period of the laboratory procedure.

FPDs were made in dental laboratories on full arch stone casts, which were isolated with separating agent. The fiber framework consisted of manual resin wetting requiring unidirectional pre-impregnated glass-fiber bundles (Stick ${ }^{\mathrm{TM}}$, Stick Tech Ltd., Finland). Each bundle consists of about 4000 glass fibers, with a diameter of $17 \mu \mathrm{m}$, embedded in a porous PMMA matrix. Glass-fiber reinforcements were manually impregnated with BisGMA-TEGDMA based light polymerizing monomer resin (Stick Resin, Stick Tech Ltd., Finland) to form a PMMA-dimethacrylate semi-inter polymer network (IPN) $[12,13]$.

Before the fibers were placed on the cast, a thin layer of flowable composite was applied at the retainer area, which was not light-cured upon placement of the fiber bundle. After light polymerization, the framework was veneered with composite resin (in Turku and Umeå: Sinfony (3M ESPE, Germany); in Nijmegen: Artglass (Hereaus Kulzer, Germany)). The composite resin was built incrementally using a heat-light polymerization oven (in Turku and Umeå the 3M ESPE oven; in Nijmegen the Heraflash).

In the second treatment session, provisional restorations were removed and the abutment teeth were cleaned from debris. In most cases the fit of the FPD was checked using a silicon material (Fit Checker, GC, Japan); if needed the fit was adapted using diamond burs. Rubberdam was used in Nijmegen only, in about $50 \%$ of the cases. The bonding surface of the FPD was treated with the monomer resin. The resin was left unpolymerized, shielded from light, for at least $3 \mathrm{~min}$ to allow the resin to penetrate and activate the semiIPN-phase of the polymethylmethacrylate polymer matrix of the FRC framework. FPDs were luted with resin composite cement (Turku and Umeå: Compolute (3M ESPE, Germany) and Variolink (Ivoclar Vivadent, Liechtenstein); in Nijmegen: Twinlook (Hereaus Kulzer, Germany) and Panavia F (Kuraray, Japan)) according to the manufacturer's instructions. After removal of excess material, the resin composite cement was light cured for 20 s per surface. After polymerization, restoration margins were finished. Occlusion was adjusted with fine diamond-burs and the restoration was polished using rubbers and polishing discs. Patients received individual instructions to maintain plaque control.

\subsection{Evaluation}

For specific evaluation of the FRC FPDs, the majority of patients were invited for a check-up once a year, up to 5 years at minimum. Besides these check-ups, patients were advised to contact the dentist from the university clinic in case an event occurred concerning their FPD. The performance of the 


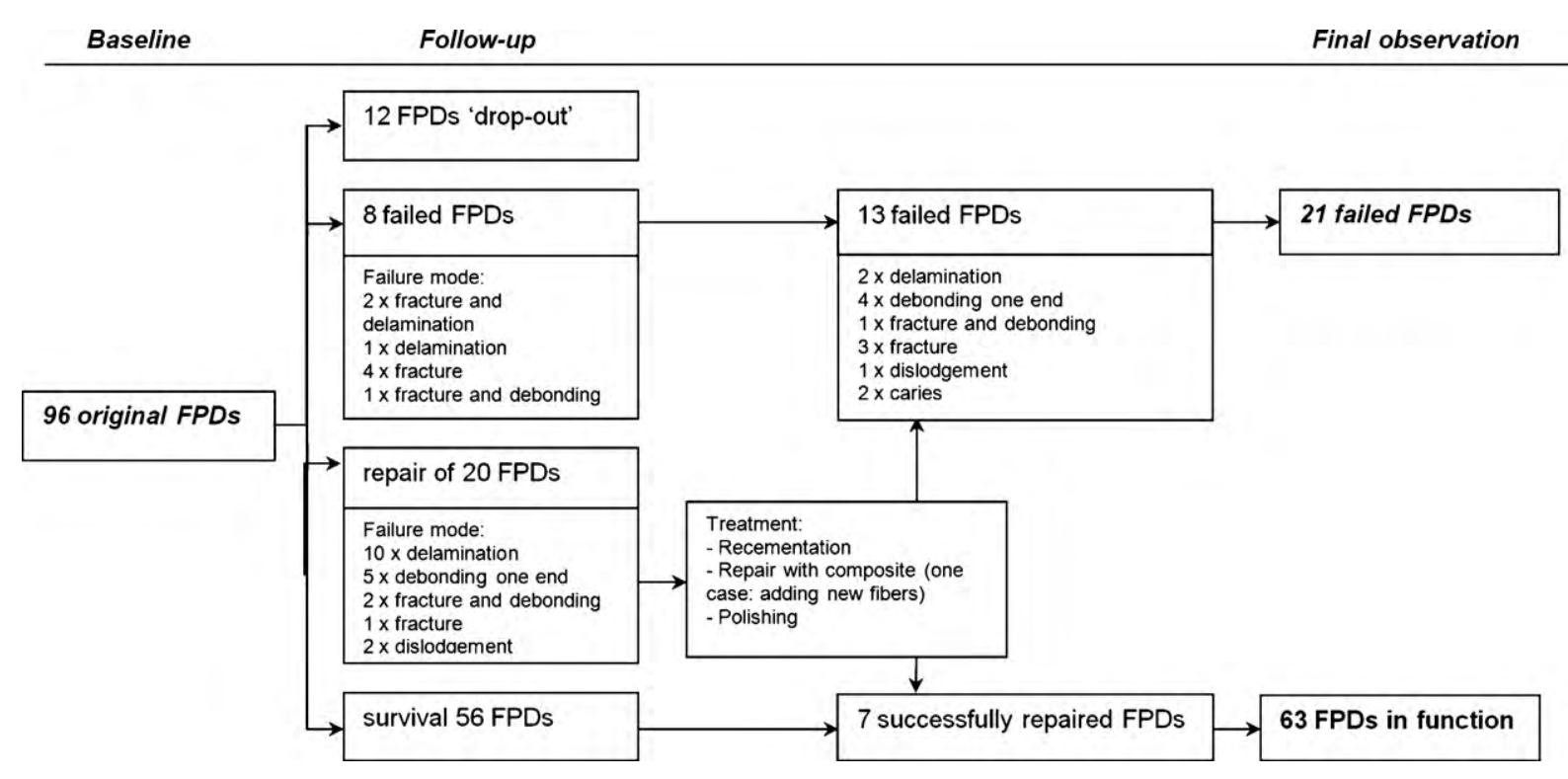

Fig. 2 - Lifecycle of posterior FRC FPDs during the follow-up period (4.5-8.9 years).

restorations was evaluated by clinical examination. Caries and periodontal status, wear of the restoration, discoloration, fractures and dislodgements were recorded. During the years 2005-2007 all patients with FPDs that were at least 4.5 years old and whose records did not already indicate a failure or removal of the restoration, were invited to participate in a clinical examination.

During the follow-up period, all interventions were recorded. Interventions may vary from finishing in case of chip fractures through repair by adding resin composite to renewal of the restoration. When records indicated interventions, the date and type of repair were recorded. If FPDs were repaired more than once, the first date of repair was used. The FPDs that could be rebonded after dislodgement were rebonded using the same procedure as had been used originally. Modes of failure were recorded as: (1) fracture of framework; (2) debonding one end; (3) dislodgement; (4) delamination of the veneering composite; (5) combination of problems. Fracture of the pontic, while the framework was still intact, was recorded as delamination.

\subsection{Analysis}

All restorations were included as individual cases. Two failure categorizations were used:

Repaired needed: Includes interventions, such as polishing and finishing after chipping of small fragments of the veneering resin composite, repair of small delaminations with restorative resin composite, or adding fibers at the connector area of the fiber framework, during follow-up. Also rebonding of FPD after dislodgement or debonding of one retainer was considered a repair.

Failure occurred: An FPD was considered failed, when problems, such as fracture of the restoration, unreparable delamination of the veneering resin composite, and combination of problems, that could not be repaired with the FPD in situ, occurred during follow-up.
Survival was analyzed at different levels: on the level of 'success' and on the level of 'survival' using the two criteria of failure as endpoints. In both cases, restorations not meeting the criterion of failure at the end of the observation period were labeled "censored". Reasons for drop-out were traced.

Kaplan-Meier survival analyses were done for the complete group of FPDs and discriminated according to retainer type and preparation form. The $95 \%$ confidence intervals for survival probability at 5 years were calculated. Correlations between variables were crosschecked and possibilities for Cox regression analyses were omitted because there are two many variables. The analyses were performed with SPSS version 16.0 (SPSS Inc., Chigaco, IL, USA).

\section{Results}

Mean follow-up time was 5.5 years, with a minimum of 4.5 years and 8.9 years as the maximum. During the follow-up period 11 patients with 12 FPDs were lost to follow-up (12.5\%). These drop-out patients could not be contacted or were not able to participate in follow-up examination mostly because of travel distance. The lifecycle of the FRC FPDs included in this study is shown in Fig. 2. Twenty-eight FPDs failed because of fracture, delamination or debonding. The operators regarded 20 of them as reparable. Failures occurred at a mean follow-up of 18-24 months (reparable and non-reparable failures, respectively). The rebonded or repaired FPDs failed again in five cases within 2-40 months.

The percentage distribution of failure modes is shown in Fig. 3. For reparable failures, delamination and debonding of one retainer-end were the main problems $52 \%$ and $28 \%$, respectively). Fracture of the framework and delamination were the main causes for failure (38\% and $20 \%$, respectively). One FPD was replaced because of caries in the abutment tooth. Combined problems included a case showing delamination and fracture of the pontic area (failure) and in three other cases 


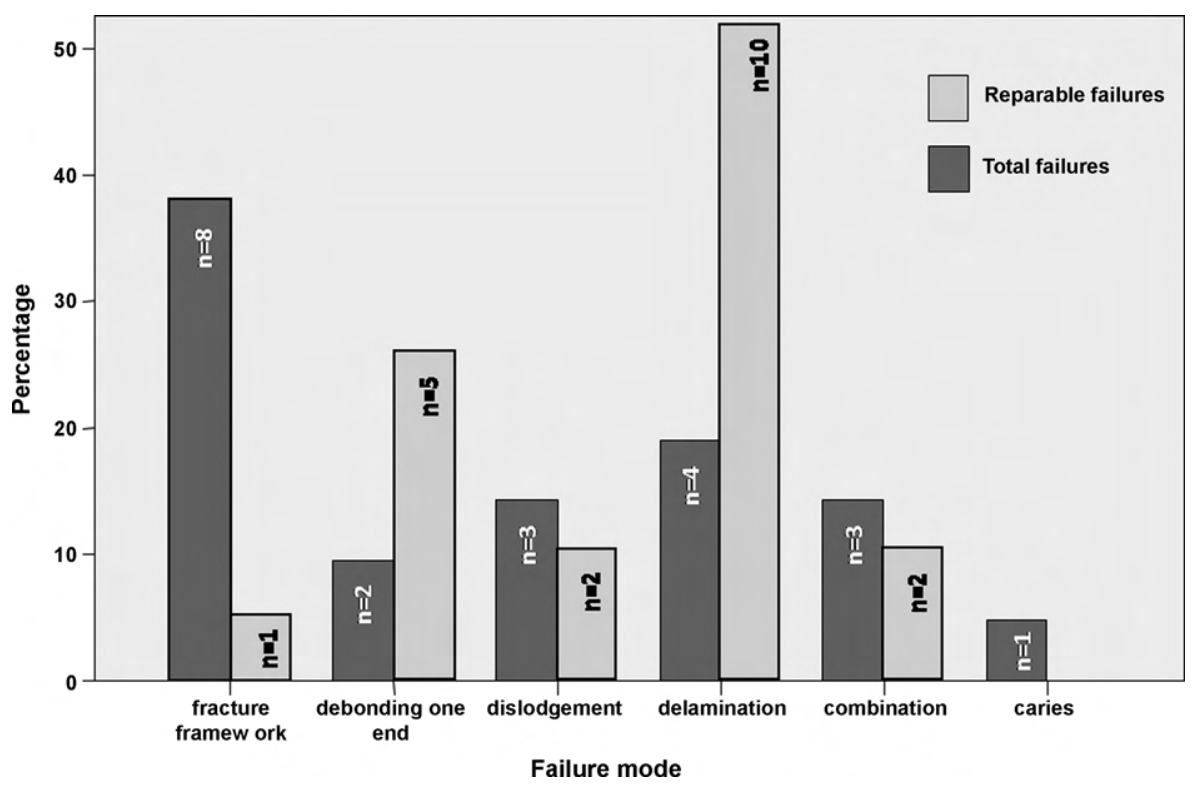

Fig. 3 - Failure mode in categories at repair and at total failure.

a combination of debonding and fracture of the retainer (one failure, two repaired). Only FPDs with surface retainers showed debonding.

Survival curves for 'success' and 'survival' up to 5 years are shown in Fig. 4. Kaplan-Meier survival rate at 5 years was $71.2 \%$ (SE 4.8\%) for success and 77.5\% (SE 4.4\%) for survival. Although survival rates for 'survival' at 5 years seemed to be higher for inlay retained FPDs in comparison with surface and hybrid FPDs ( $82 \%$ vs. $78 \%$ and $66 \%$ ), survival rates for different groups (surface vs. hybrid vs. inlay) were not significantly
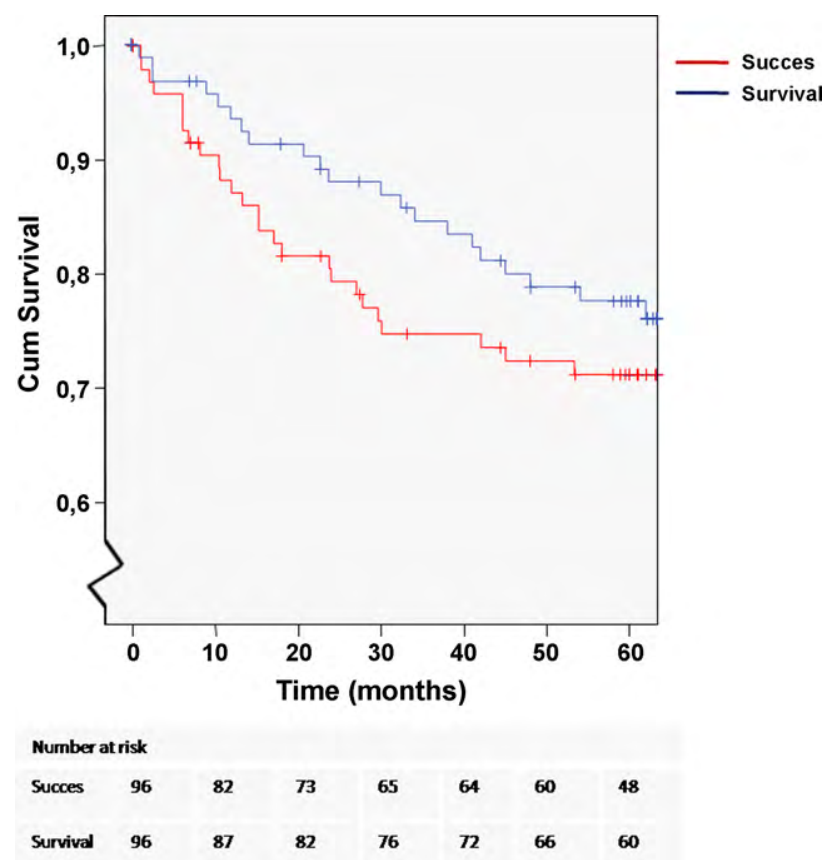

Fig. 4 - Restoration survival probability as a function of time for posterior FRC FPDs $(\boldsymbol{n}=96)$. different for both 'success' and 'survival' (Fig. 5; log rank test $p>0.05)$.

The two veneering materials were exclusively related to the different institutes. Therefore, analyses on the survival rates for institutes or material were not feasible. In addition, interaction between independent variables such as operator, patient age, preparation, type of FPD and luting cement hampers a valid regression analysis.

\section{Discussion}

This study reports clinical follow-up data on three-unit posterior indirect FRC FPDs after a mean service time of 5.5 years. This study forms part of a trial including the previously referred anterior FPDs. A survival rate of $78 \%$ was observed for posterior FPDs, which is higher than the $63 \%$ survival rate we found for anterior FRC FPDs after 5 years [11]. Thus, our hypothesis is rejected. Other published clinical studies on FRC FPDs do not discriminate between anterior or posterior bridges and survival rates of 75-95\%, after shorter follow-up times of 3-4 years, have been reported. A study using similar (manual resin impregnation requiring) FRC material, but mixed FPD designs, demonstrated a survival rate of $93 \%$ after 3.5 years [14]. Given the longer follow-up time of our study, the present result seems to be in line with the abovementioned survival rates.

The trial as a whole was a mix of a prospective trial and a retrospective evaluation. The strict protocol of a randomized clinical trial could not be maintained, but generally accepted limitations of retrospective studies, like their nonprotocolized design, are not applicable to this study. Operators worked according to a clinical protocol and the restoration design was restricted to three-unit FPDs. However, it was not possible to assign patients and type of retainers on a random basis and also the three clinical centers differed on details concerning clinical and technical procedures. These differ- 

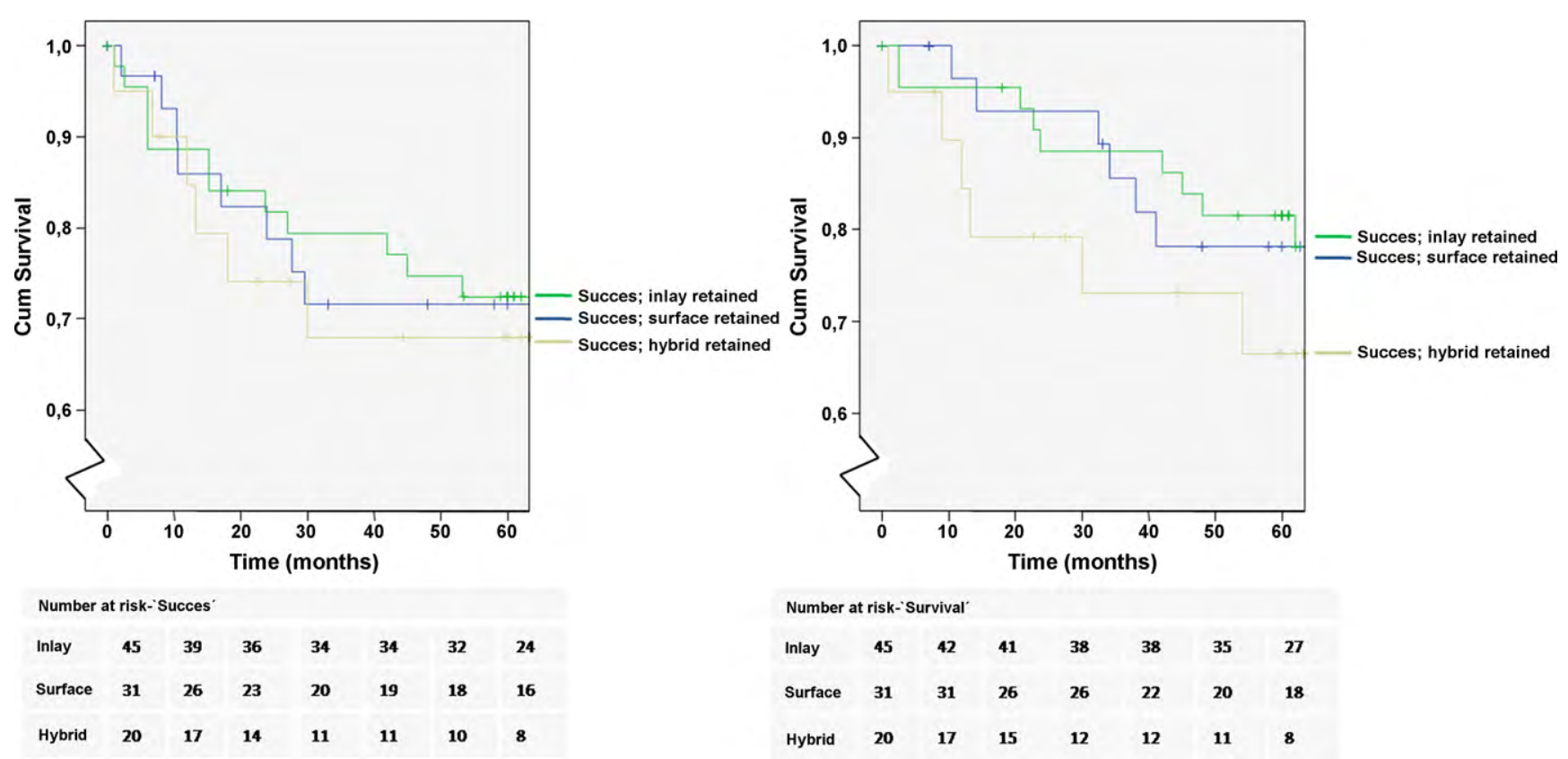

Fig. 5 - Restoration survival probability as a function of time for surface FPDs $(n=31)$, inlay FPDs $(n=45)$ and hybrid FPDs $(n=20)$ for 'success' (right) and 'survival' (left).

ences complicated analyses and prevented firm conclusions on items of interest. On the other hand it gave us the opportunity to obtain indications of the clinical performance of FRC FPDs with small differences in design. During analyses it appeared that survival results of one operator in this study substantially differed from the others. The slope of the survival curve of the FPDs made by this operator differed, and this could not be explained by design, material, or dentist factors. Possibly, differences in case selection could be the reason. If this operator is excluded from the analysis the survival rate would increase to $84 \%$.

Striking is the difference in survival of three-unit FRC FPDs in the anterior compared to the posterior area. To our knowledge, no other study on FRC bridge constructions has been published that could confirm or refute such a difference. The difference between survival of anterior and posterior FRC FPDs can be traced to a difference in volume of the constructions. The retainers of anterior FPDs are thin and micro-cracks in the veneering composite layer can easily occur, followed by further degradation of the veneer. The volume of composite on top of the fiber frame of posterior bridges is generally much higher and the bulk of material prevents early crack forming. Indeed, we found relatively more delaminations with anterior than with posterior FPDs. Furthermore, it had been stated that the weakest part of a bridge construction is the connector area $[15,16]$. For anterior FPDs the connector area is relatively thin compared to the connector area in a posterior FPD. Moreover, loading of posterior bridges is expected to be of vertical angulation with lower change of rotation forces compared to anterior bridges. Given the volume difference, the anterior bridge has lower opportunity to withstand these occlusal loading forces.

When studying metallic resin bonded FPDs with retainers of the Maryland design with minimal, strategic preparations, it was found that anterior FPDs survived better than posterior FPDs $[17,18]$. Considering the high survival rate in the anterior region compared to posterior, Creugers did not recommend to prepare abutment teeth extensively. In the present study, the difference in preparation of abutment teeth between anterior and posterior is expected to influence the survival as main difference in design. It can be assumed that preparation for anterior FRC FPDs thus is recommended.

A trend towards better survival of inlay-retained FPDs over other FPD designs was observed. Similar observations can be found in the literature [14]. An inlay retainer of sufficient volume $(2 \mathrm{~mm} \times 2 \mathrm{~mm} \times 2 \mathrm{~mm})$ seems to provide sufficient resistance against rotational forces when it can be adhesively retained to tooth tissues. Although surface retention may offer even more resistance against rotational and oblique detaching forces on the condition that the retainer is provided with axial support for example an occlusal rest, the volume problem as outlined in the previous paragraph may here also be of importance to the formation of cracks, and finally failure of the bridge.

Two different veneering composites were used while manufacturing the FPDs, namely Artglass and Sinfony. Conclusions towards the behavior of materials could not be drawn from the results in this study, because of the strong correlation between materials, institutes and FPD design. Compared to laboratory composites it is described that Artglass has lower mechanical properties in terms of fracture, tensile, compressive, and flexural strength [19]. However, the material properties of both composites do not deviate to a great extent and their behavior should be quite comparable.

Veneering composite fractures, i.e. delaminations constituted the mode of failure most commonly observed. This is most likely a result of insufficient support for the pontic area offered by the solely unidirectional framework fibers 
as applied in our study. Clinical reports demonstrated an improved resistance against veneering composite fractures of a larger substructure volume at the pontic area by using a wrap around design, or a bundle of fibers oriented perpendicularly towards longitudinal fibers $[10,20,21]$. We furthermore found fractures of the fiber framework. Like the anterior FRC FDPS of this trial, the low fiber volume fraction of a manually impregnated composite may result in insufficient strength of the material. Therefore, advice is to apply more than one fiber bundle in the framework, additional reinforcement at the pontic area, and to provide sufficient volume of composite at the retainer and connector sites.

All in all it can be stated that, considering the tissue saving characteristics, relatively low costs and tooth colored material, these kind of restorations are an interesting (semi) permanent solution. The results in this study suggests that the application of FRC FPDs in the posterior region can be a good alternative, especially in cases of young patients where implant therapy is not (yet) indicated.

\section{Conclusion}

In the present study, three unit posterior FRC FDPs demonstrated a success rate of $71 \%$ during an observation period of 4.5-8.9 years. If repaired FPDs were included as successful performing constructions, the survival rate was $78 \%$. Survival rates of inlay, hybrid and surface retained FPDs did not significantly differ. Delamination, debonding and fracture of the framework were most prevalent failure modes and debonding was seen only for surface retained FPDs.

\section{Acknowledgements}

This study was supported in part by the County Council of Västerbotten and The Swedish National Board of Health and Welfare.

\section{REFERENCES}

[1] Pjetursson BE. A systematic review of the survival and complication rates of resin-bonded bridges after an observation period of at least 5 years. Clin Oral Impl Res 2008;19:131-41.

[2] Probster B, Henrich G. 11-Year follow-up study of resin-bonded fixed partial dentures. Int J Prosthodont 1997;10:259-68.

[3] Vallittu PK. Flexural properties of acrylic resin polymers reinforced with unidirectional and woven glass fibers. J Prosthet Dent 1999;81:318-26.

[4] Fennis WM, Tezvergil A, Kuijs RH, Lassila LV, Kreulen CM, Creugers $\mathrm{NH}$, et al. In vitro fracture resistance of fiber reinforced cusp-replacing composite restorations. Dent Mater 2005;21:565-72.

[5] Xu HH, Schumacher GE, Eichmiller FC, Peterson RC, Antonucci JM, Mueller HJ. Continuous fiber preform reinforcement of dental resin composite restorations. Dent Mater 2003;19:523-30.

[6] Jokstad A, Gokce M, Hjortsjo C. A systematic review of the scientific documentation of fixed partial dentures made from fiber-reinforced polymer to replace missing teeth. Int J Prosthodont 2005;18:489-96.

[7] van Heumen CCM, Kreulen CM, Creugers NHJ. Clinical studies of fiber-reinforced resin bonded FPDs: systematic review. Eur J Oral Sci 2009;117:1-6.

[8] Behr M, Rosentritt M, Handel G. Fiber-reinforced composite crowns and FPDs: a clinical report. Int J Prosthodont 2003;16:239-43.

[9] Göhring TN, Schmidlin PR, Lutz F. Two-year clinical and SEM evaluation of glass fiber-reinforced inlay fixed partial dentures. Am J Dent 2002;15:35-40.

[10] Monaco C, Ferrari M, Miceli GP, Scotti R. Clinical evaluation of fiber-reinforced composite inlay FPDs. Int J Prosthodont 2003;16:319-25.

[11] van Heumen CCM, van Dijken JW, Tanner J, Pikaar R, Lassila LVJ, Creugers NHJ, et al. Five-year survival of 3-unit fiber-reinforced composite fixed partial dentures in the anterior area. Dent Mater 2009;25:820-7.

[12] Lastumäki TM, Lassila LVJ, Vallittu PK. The semi-interpenetrating polymer network matrix of fiber-reinforced composite and its effect on the surface adhesive properties. J Mater Sci: Mater Med 2003;14: 803-9.

[13] Vallittu PK. Interpenetrating polymer networks (IPNs) in dental polymers and composites. J Adhes Sci Technol 2009;23:961-72.

[14] Vallittu PK. Survival rates of resin-bonded, glass fiber-reinforced composite fixed partial dentures with a mean follow-up of 42 months: a pilot study. J Prosthet Dent 2004;91:241-6.

[15] Rappelli G, Scalise L, Procaccini M, Tomasini EP. Stress distribution in fiber-reinforced composite inlay fixed partial dentures. J Prosthet Dent 2005;93:425-32.

[16] Rosentritt M, Behr M, Lang R, Handel G. Experimental design of FPD made of all-ceramics and fibre-reinforced composite. Dent Mater 2000;16:159-65.

[17] Creugers NHJ, Kayser AF. An analysis of multiple failures of resin-bonded bridges. J Dent 1992;20:348-51.

[18] Creugers NHJ, Kayser AF, van't Hof MA. A seven-and-a-half-year survival study of resin bonded bridges. J Dent Res 1992;71:1822-5.

[19] Hamakubo Y, Sawase T, Yoshida K, Kamada K, Taira Y, Atsuta $\mathrm{M}$. The physical properties of a machinable resin composite for esthetic restorations. Dent Mater J 2005;241:24-9.

[20] Freilich MA, Meiers JC, Duncan JP, Eckrote KA, Goldberg AJ. Clinical evaluation of fiber-reinforced fixed bridges. J Am Dent Assoc 2002;133:1524-34.

[21] Xie Q. Comparison of load-bearing capacity of direct resin-bonded fiber-reinforced composite FPDs with four framework designs. J Dent 2007;35:578-82. 